\title{
Deaf and Hard-of-hearing Individuals' Preferences for Wearable and Mobile Sound Awareness Technologies
}

\author{
Leah Findlater \\ University of Washington \\ Seattle, WA \\ leahkf@uw.edu \\ Jon Froehlich \\ University of Washington \\ Seattle, WA \\ jonf@cs.uw.edu
}

\author{
Bonnie Chinh \\ University of Washington \\ Seattle, WA \\ bchinh@uw.edu \\ Raja Kushalnagar \\ Gallaudet University \\ Washington, DC \\ raja.kushalnagar@gallaudet.edu
}

\author{
Dhruv Jain \\ University of Washington \\ Seattle, WA \\ djain@cs.washington.edu
}

Angela Carey Lin

University of Washington

Seattle, WA

\begin{abstract}
To investigate preferences for mobile and wearable sound awareness systems, we conducted an online survey with 201 $\mathrm{DHH}$ participants. The survey explores how demographic factors affect perceptions of sound awareness technologies, gauges interest in specific sounds and sound characteristics, solicits reactions to three design scenarios (smartphone, smartwatch, head-mounted display) and two output modalities (visual, haptic), and probes issues related to social context of use. While most participants were highly interested in being aware of sounds, this interest was modulated by communication preference-that is, for sign or oral communication or both. Almost all participants wanted both visual and haptic feedback and $75 \%$ preferred to have that feedback on separate devices (e.g., haptic on smartwatch, visual on head-mounted display). Other findings related to sound type, full captions vs. keywords, sound filtering, notification styles, and social context provide direct guidance for the design of future mobile and wearable sound awareness systems.
\end{abstract}

\section{CCS CONCEPTS}

\section{- Human-centered computing $\rightarrow$ Empirical studies in} accessibility.

Permission to make digital or hard copies of all or part of this work for personal or classroom use is granted without fee provided that copies are not made or distributed for profit or commercial advantage and that copies bear this notice and the full citation on the first page. Copyrights for components of this work owned by others than ACM must be honored. Abstracting with credit is permitted. To copy otherwise, or republish, to post on servers or to redistribute to lists, requires prior specific permission and/or a fee. Request permissions from permissions@acm.org.

CHI 2019, May 4-9, 2019, Glasgow, Scotland UK

(C) 2019 Association for Computing Machinery.

ACM ISBN 978-1-4503-5970-2/19/05 ..\$15.00

https://doi.org/10.1145/3290605.3300276

\section{KEYWORDS}

Deaf, hard of hearing, hearing loss, sound awareness, mobile, wearable, online survey, user study

\section{ACM Reference Format:}

Leah Findlater, Bonnie Chinh, Dhruv Jain, Jon Froehlich, Raja Kushalnagar, and Angela Carey Lin. 2019. Deaf and Hard-of-hearing Individuals' Preferences for Wearable and Mobile Sound Awareness Technologies. In CHI Conference on Human Factors in Computing Systems Proceedings (CHI 2019), May 4-9, 2019, Glasgow, Scotland UK. ACM, New York, NY, USA, 13 pages. https://doi.org/10.1145/ 3290605.3300276

\section{INTRODUCTION}

Sound awareness has wide-ranging impacts for persons who are deaf or hard of hearing (DHH), from being notified of safety-critical information like a ringing fire alarm to more mundane but still useful sounds like the clothes dryer ending a cycle [27]. While hearing aids and surgically implanted devices can improve sound and speech recognition, they do not eliminate hearing loss; residual issues can include speech intelligibility, ability to interpret sound direction, sensitivity to background noise, or in the case of directional hearing aids, missed noises to the side and back of the wearer [5]. The success of these devices also depends on a number of factors, such as the wearer's level of hearing loss, linguistic abilities, and, in the case of cochlear implants, therapy to learn (or relearn) the sense of hearing [32].

Motivated by these limitations and to complement existing sound awareness strategies, researchers have investigated systems to sense and feed back speech and non-speech sounds to DHH users. Early work by Matthews et al. [27] examined sound awareness needs across a variety of contexts (at home, at work, while mobile), and built and evaluated a 
proof-of-concept prototype to display office sounds on a computer monitor. More recent solutions have begun to investigate other form factors, including head-mounted displays for speech captioning $[16,17,34]$, wrist-worn or smartwatch displays [20,29], and smartphone apps for general sound detection [3, 30, 39]. While formative studies and, in some cases, evaluations of these technologies have yielded useful insights, the studies tend to be qualitative and/or focused on a single device form factor and have not been designed to examine issues around social acceptability.

To investigate $\mathrm{DHH}$ individuals' preferences for mobile and wearable sound awareness systems across a range of design variables, we report on an online survey of 201 participants. The survey explores how demographic factors may affect perceptions of wearable technologies (e.g., age, gender), examines interest in the idea of sound awareness technology as well as in specific sounds and sound characteristics, solicits reactions to three sound feedback scenarios (smartphone, smartwatch, head-mounted display (HMD)) and to visual and haptic feedback, and investigates perceived utility and social comfort.

Our findings show that while most participants were "very" or "extremely" interested in being aware of sounds, this interest was modulated by communication preference-that is, preference for sign or oral communication or both. We provide a prioritization of sound types and sound characteristics (e.g., urgent alerts and voices directed at you are considered of highest interest), which extends past work [3, 27, 39] with quantitative and/or more detailed results. Further, we find that smartwatches are the most preferred individual form factor, but $92 \%$ of participants wanted both visual and haptic feedback and $75 \%$ of participants wanted that feedback to be provided on separate devices (haptic on smartwatch, visual on smartphone or HMD). Other findings relate to full captions vs. keywords, sound filtering, notification styles, and social context provide direct guidance for the design of future mobile and wearable sound awareness systems.

The contributions of this paper include: (1) extension of findings on sound awareness interest [3, 27, 39] to include influences of demographic factors; (2) characterization of preferences for form factor, output modality, and speech vs. non-speech sounds; (3) comparison of subjective utility and social comfort related to these form factors and feedback modalities; (4) design recommendations for future mobile or wearable sound awareness technologies.

\section{RELATED WORK}

Our work builds on research in the sound awareness needs of DHH users, visual and tactile approaches to sound awareness, and the social acceptability of wearable and assistive technologies.

\section{Sound Awareness Needs and Behaviors}

Understanding sound awareness needs from the DHH community is important for informing technology design. To converse with partners using spoken language, people with hearing loss use a variety of strategies, including gestures and two-way note taking [10]. Speechreading combines body language, facial expressions, situational cues, and any auditory input that is available to understand parts of oral conversation [24]. Sound awareness needs for oral conversation range from knowing who is speaking to a desire for real-time captioning [16].

In early work on non-speech sound awareness needs, Matthews et al. [27] conducted two interview studies with a total of $18 \mathrm{DHH}$ participants to assess needs across three contexts: home, work, and while mobile. Their findings highlight the need to be aware of other people and their activities, the importance of sound awareness while outside the home, and the desire for end-user customization of sound information. While safety-related sounds were generally of interest (e.g., alarms), awareness needs varied across contexts, such as home (e.g., doorbells), transit (e.g., honking), and work (e.g., activities of coworkers). Jain et al. [18] have also more recently examined home sound awareness needs in depth via interview studies with $\mathrm{DHH}$ participants.

More recently, in a survey with $87 \mathrm{DHH}$ participants, Bragg et al. [3] confirmed many of these older findings and showed that hard of hearing users may be more interested than deaf users in some types of sounds (e.g., phone ringing, nearby conversations), and that some sound characteristics (identity, location, urgency, and confidence) are more important than others. However, those conclusions are not based on reported statistically significant differences.

Finally, Sicong et al. [39] surveyed $60 \mathrm{DHH}$ youth and adults aged 10-26 on their sound awareness needs, finding that the primary sounds of interest were related to social interactions (e.g., door knock, presence of others) and "early warning events" (e.g., microwave beep, fire alarm). Unlike the other studies, Sicong et al. also provided an initial examination of form factors. They found a preference for a smartphone over a "head-mounted tool", though participants were not given a description of what was meant by a headmounted tool.

Our survey extends these past studies by employing a larger sample size to quantify the impacts of user background characteristics on sound interest. We also assess sound awareness preferences and social acceptability issues with different mobile and wearable devices and examine specific design issues that arise with a continuous sound recognition system (e.g., filtering, push vs. pull notification). 


\section{Visual and Haptic Sound Awareness Approaches}

Visual and haptic modalities have been used for sound awareness. Most visual solutions focus on non-speech sounds and non-wearable solutions [12, 27, 28, 39, 41]. For example, Matthews et al. [27] studied sound visualizations projected on a desktop or office wall and found that participants preferred designs that were easy to interpret, glanceable, and "appropriately distracting."

As mentioned in the Introduction, a small number of projects have begun exploring wearable visual solutions, including wrist-worn displays [20, 29] and HMDs [9, 17, 22, $34,37]$. HMDs have also been used to display pre-recorded captions for moviegoers [40] and pre-recorded sign language interpretations in educational environments $[11,19,33]$. Offthe-shelf text-to-speech functionality on smartphones can also support communication between deaf and hearing persons, although important challenges with speech recognition accuracy exist, particularly with recognizing deaf speech [8]. While this work illustrates the increasing interest in mobile and wearable sound awareness, studies to date have only briefly $[17,39]$ compared form factors and design options, which our survey does in more depth.

Haptic feedback has also been used to translate and convey acoustic properties for DHH users [6, 44, 45], such as for simple sound notification [29], to convey sound direction via waist-mounted vibro-motors [4], or to supplement visual captions [23]. For example, Yeung et al. [44] created a tactile display that transformed pitch information into a 16-channel vibro-pattern on the forearm. While positive outcomes have been shown-e.g., in the perceptual enhancement of words and phonemes among lipreaders [6]-tactile devices remain an active area of research and, compared to visual approaches, offer much lower information throughput. However, some preliminary work suggests that haptic feedback may be useful for notifying the user that a sound has occurred before more detail is presented visually [29]. Our survey further probes users' preferences for visual vs. vibrational feedback, and investigates how preferences vary across sound types and form factors.

\section{Social Issues of Wearables and Assistive Technology}

Important issues of social acceptability arise with wearable devices and technologies that are perceived to be "assistive." While norms are evolving, wearable device acceptability can be modeled as a tension between aspirational desires (e.g., positive reflection of one's self-image, utility) and negative social impacts (e.g., privacy concerns, distraction) [21]. Other factors shown to impact acceptability include gender [36], functionality [2], and placement of the device [36, 42]. Amid these concerns, assistive technologies designed to increase inclusion can also reinforce the social distancing of people with disabilities, as the assistive technology can emphasize the presence of difference [25] and contribute to stigma [38]; however, onlookers may be more accepting of a wearable device if they perceive it to be assistive [35]. In Deaf culture, deafness is not considered to be a disability as aural communication is not used. Deaf people and hearing peers will be frustrated with a design that does not respect visual prioritization and ordering for mixed communication and notification. Inappropriate designs can increase communication friction and elevate risk of misunderstanding social and communicative intent, leading to increased social distance [1]. Motivated by these complex concerns, we investigate social acceptability across a variety of contexts.

\section{METHOD}

To understand user needs for a mobile or wearable sound awareness system, we conducted an online survey with $\mathrm{DHH}$ participants. The survey included questions on hearing loss, interest in sound awareness (including sound types and sound characteristics), preferences for different form factors and feedback modalities, and issues of social acceptability.

\section{Hosting Platform and Recruitment}

The survey was hosted on SurveyMonkey and was designed to take up to 20 minutes. Upon completion, participants could opt into a drawing for a \$50 Amazon gift certificate. Participants were recruited through $\mathrm{DHH}$ organizations in the United States, social media, and word of mouth.

\section{Survey Outline}

Following an initial screening question ("Are you deaf or hard of hearing?"), the survey included 34 open- and closed-form questions, grouped as follows (see Supplementary Material):

Demographics: included age, gender, hearing loss level, preferred in-person communication methods (sign language, oral, etc.), and current sound awareness strategies.

Interest in sound awareness: assessed interest in sound awareness, current technologies to support sound awareness, interest in specific types of sounds (e.g., outdoor mechanical sounds, indoor mechanical sounds, voices), and interest in different sound characteristics (e.g., loudness, duration).

Imagining device designs: presented mobile and wearable sound awareness device scenarios to solicit reactions and compare three form factors (smartphone, smartwatch, and HMD). Figure 1 shows the visual scenarios, which were presented along with the following textual description:

The following sketches show three different devices: smartphone, smartwatch, and head-mounted display. Imagine that each device has the ability to constantly monitor and identify the sounds around you, and to inform you about those sounds, 


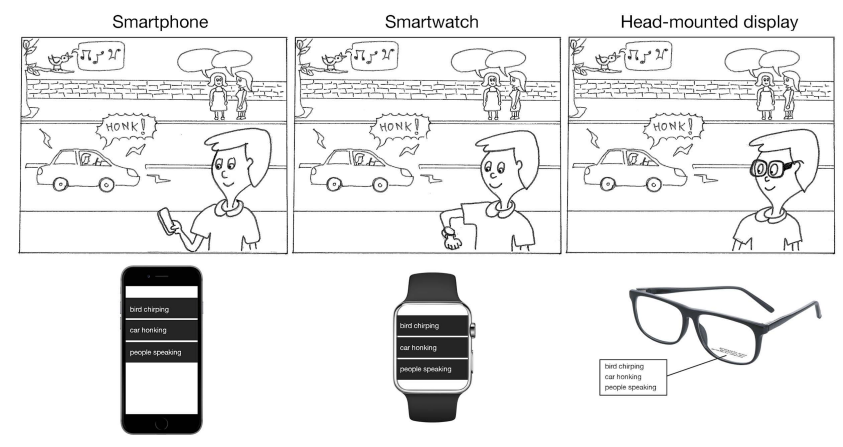

Figure 1: Mobile and wearable device scenarios shown to participants, designed to probe reactions to three form factors: smartphone, smartwatch, and head-mounted display.

either through visual or vibrational feedback. Examples include, a visual popup or vibration could occur when an important sound is detected, or the device could provide you with an overview of the current sounds around you.

Oral conversation: asked about preferences for features that support oral conversation, such as captions.

Visual and vibrational feedback: asked about utility and application of these two types of feedback as well as preferences for how to provide the feedback.

Filtering and notification: examined whether some sounds should be filtered out and/or implemented via push vs. pull notifications.

Social context: collected comparative data on the utility and social comfort of using these devices across a variety of social contexts (by yourself, with family or close friends, with work colleagues, with strangers).

The survey closed with the opportunity to provide additional comments about the technologies proposed or the survey. All participants completed survey questions in the same order, but answer options for sound types and form factors were randomized automatically by the survey software.

\section{Data and Analysis}

There were 207 completed responses. An additional $44 \mathrm{DHH}$ participants began the survey but did not complete it, resulting in a dropout rate of $17.5 \%$ (44/251). The median survey completion time was 16.6 minutes.

For open-form questions, we employed an iterative coding process [15]. For each question, two researchers developed an initial codebook with input from other research team members, then independently applied the codebook to a random subset of 40 responses (or fewer if there were fewer than 40 responses). Cohen's kappa was calculated to assess interrater reliability for each code and those with low kappa values were updated (clarified, removed, or merged). This process was repeated until almost all data had been coded once ( 5 iterations $\mathrm{x} 40$ responses $=200 / 201$ responses coded). At that point, the codebook was considered final, with an average kappa of $0.79(S D=0.06)$ and raw agreement of $0.93(S D=.05)$ across all codes reported in this paper. All codes were finalized, with disagreements resolved through consensus between two research team members.

To analyze the quantitative data, we provide descriptive statistics (e.g., frequencies, medians). For our primary ordinal rating scale measures, we use non-parametric statistical tests, including ordinal logistic regression using the clm function in R's ordinal package, and ANOVAs with aligned rank transform (a non-parametric alternative to ANOVAs [43]). We use Wilcoxon and Mann Whitney $U$ tests for posthoc pairwise comparisons of the ANOVAs with ART, along with Holm-Bonferroni corrections [14].

For quantitative analyses, we often include a breakdown based on participants' preferred in-person communication method, which we show is significantly related to overall interest in sound awareness. In the survey, this question had the following options: sign language, oral (spoken) communication, writing, other (fill in the blank); participants could select more than one option. Almost all participants (201/207) chose at least sign and/or oral communication; the remaining six participants chose only writing or wrote in 'other'; we exclude these six participants from our analysis.

\section{RESULTS}

We report on survey responses from the $201 \mathrm{DHH}$ participants who completed the survey and specified a preference for sign language, oral communication, or both.

\section{Participant Background Characteristics}

Participants ranged from 20 to 94 years old, with a median age of $42(M=47.4, S D=18.6)$. The majority $(55.2 \%)$ identified as female $(N=111)$, while $42.3 \%(N=85)$ identified as male, $2.0 \%(N=4)$ as non-binary, and one person $(0.5 \%)$ declined to answer this question. Table 1 shows self-reported level of hearing loss in the participant's "best" ear, with most participants reporting profound or severe hearing loss. Similarly, most participants $(85.1 \%, N=171)$ used a hearing device: $47.8 \%(N=96)$ used a hearing aid, $24.9 \%(N=50)$ used a cochlear implant, and $12.4 \%(N=25)$ used both a hearing aid and a cochlear implant. When asked how they preferred to communicate with others, $48.8 \%$ or participants $(N=98)$ selected oral (spoken) language, 29.5\% (61) selected sign language, and 20.9\% (42) selected both oral and sign language. Most $(72.1 \%$ of 201$)$ reported being a speechreader. The survey did not distinguish if participants identified as Deaf/deaf, hard-of-hearing, or hearing. 

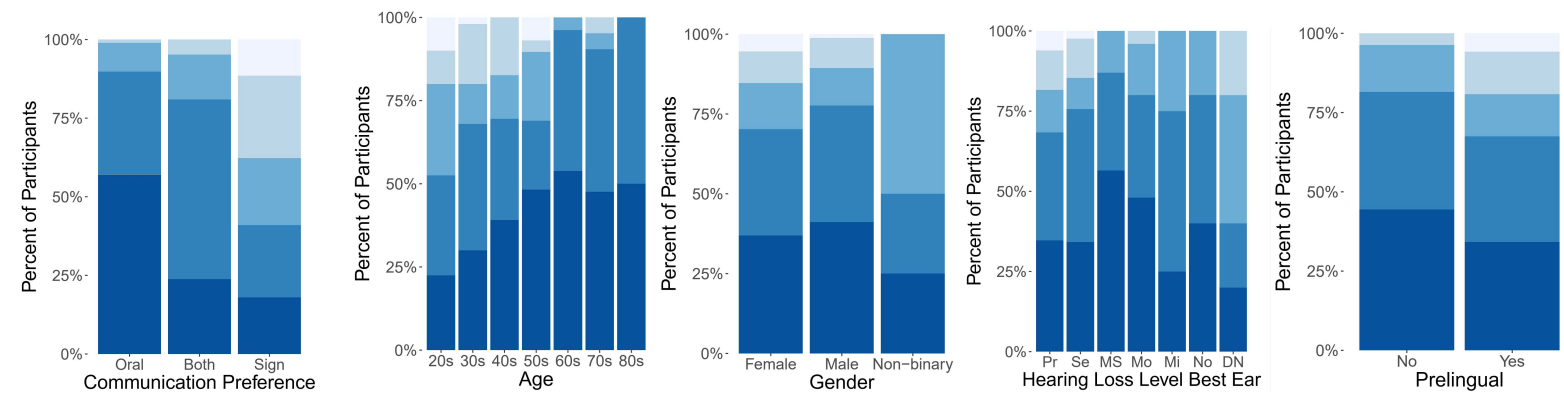

Interest Level Not interested
Slightly interested Somewhat interested
Very interested Very interested
Extremely intereste

Figure 2: Relationship between participant background characteristics and overall interest in sound awareness. Hearing loss levels: $\mathrm{Pr}=$ profound, Se=severe, MS=moderately severe, Mo=moderate, $\mathrm{Mi}=$ mild, No=none, DN=don't know. All graphs show $N=201$ except for Gender, where one participant who declined to answer this question is excluded.

Table 1: Hearing ability in best ear as reported by participants. $(N=201)$

\begin{tabular}{lcc}
\hline Hearing loss level & $\begin{array}{c}\text { Number of } \\
\text { participants }\end{array}$ & $\begin{array}{c}\text { Percent of } \\
\text { participants }\end{array}$ \\
\hline Profound: $91 \mathrm{~dB}$ or more & 98 & $48.8 \%$ \\
Severe: $71-90 \mathrm{~dB}$ & 41 & $20.4 \%$ \\
Moderately severe: $51-70 \mathrm{~dB}$ & 23 & $11.4 \%$ \\
Moderate: $31-50 \mathrm{~dB}$ & 25 & $12.4 \%$ \\
Mild: $<30 \mathrm{~dB}$ & 4 & $2.0 \%$ \\
No hearing loss & 5 & $2.5 \%$ \\
Don't know & 5 & $2.5 \%$ \\
\hline
\end{tabular}

In terms of general technology use, almost all participants $(89.6 \%, N=180)$ used a smartphone, $12.9 \%(N=26)$ used a smartwatch, and $1.5 \%(N=3)$ reported experience with an HMD such as Google Glass or Oculus Rift; an additional 9.5\% $(N=19)$ used none of these devices. Nineteen participants (9.5\%) also reported using another wearable device such as a Fitbit, or a Pocketalker amplifier. When asked about whether they use a mobile or wearable device or computer to help be aware of sounds, $26.9 \%(N=54)$ answered yes. Several of these instances included hearing aids connected to smartphones, a sound amplification system, an FM system, or additional microphone (e.g., Roger Pen). Other responses from five or more people included using text-to-speech on a smartphone to communicate with hearing people $(N=5)$ and using a smartwatch to relay vibration alerts from a smartphone $(N=5)$.

\section{Interest in Sound Types and Characteristics}

We examine overall interest in sound awareness, interest in specific types and characteristics of sound, and interest specifically in speech-either full captions or keywords.

Overall Interest in Sound Awareness. Overall interest in sound awareness was high (Figure 2), with $73.1 \%$ of participants
( $N=147)$ being "very" or "extremely" interested. When converted to a numeric scale $(1=$ "not" to 5 = "extremely interested"), the median interest was 4.0 and the average was $3.9(S D=1.1)$, indicating "very interested."

To understand how interest varies based on participant background characteristics, we also examined how overall interest varied by communication preference, age, whether hearing loss was prelingual or not, self-reported level of hearing in best ear, and gender (Figure 2).

After filtering out five participants who reported "don't know" for level of hearing loss in best ear (leaving $N=196$ ), we built an ordinal logistic regression model to identify which factors most greatly contribute to interest in sound awareness. The results are shown in Table 2, showing a significant increase in sound awareness interest for oral and for both oral+sign communicators compared to signers only. The odds ratios can be interpreted as the increased $(>1)$ or decreased $(<1)$ odds of being more interested in sound awareness compared to the baseline (for categorical variables) or for each unit change in the predictor (for continuous variables). Sign language is the baseline for communication preference, female is the baseline for gender, and yes is the baseline for prelingual hearing loss. Age and hearing loss in best ear are modeled as continuous.

The 98 participants who preferred oral communication had the highest overall interest level in sound awareness, with a median rating of "extremely interested" (on a 5-point scale: $M=4.5, S D=0.7$ ), whereas the 61 participants who preferred signing had the lowest interest level, with a median rating of "somewhat interested" $(M=3.1, S D=1.3)$. The 42 participants who preferred both signing and oral communication were in the middle in terms of interest, with a median rating of "very interested" $(M=4.0, S D=0.8)$.

None of the other independent variables were significant after controlling for communication preference. Age, which appears to be related to sound interest in Figure 2, is also highly related to communication preference: participants 
Table 2: Odds ratios of the ordered logistic regression on overall sound awareness interest. See main text for baselines and other interpretation detail. The odds ratios show that "both" and "oral" are significantly more likely to have higher interest than the baseline of "sign". $(N=196)$

\begin{tabular}{lccc}
\hline Predictor & Odds ratio & $z$-value & $p$ \\
\hline Communication - both & 4.09 & 3.60 & $<.001$ \\
Communication - oral & 10.58 & 5.69 & $<.001$ \\
Hearing loss in best ear & 0.96 & -0.38 & $\mathrm{~ns}$ \\
Prelingual hearing loss & 1.45 & 1.14 & $\mathrm{~ns}$ \\
Age & 1.01 & 1.27 & $\mathrm{~ns}$ \\
Gender - male & 1.06 & 0.20 & $\mathrm{~ns}$ \\
Gender - non-binary & 0.71 & -0.37 & $\mathrm{~ns}$ \\
\hline
\end{tabular}

who preferred oral communication were on average 57.4 years old $(S D=18.7)$, whereas those who preferred signing were $37.9(S D=12.7)$ and those who preferred both were also $37.9(S D=12.2)$. This pattern may be partly because many older adults with age-related hearing loss may not know sign language. However, trends in deaf education patterns are also related: auditory-verbal communication was dominant until the 1970s, at which point simultaneous speech and sign communication rose in popularity; in the 1990s and onwards, bilingual-bicultural communication largely supplanted the first two approaches [26, 31]. Communication preference is thus probably modulated both by hearing ability, and by educational and family environments. Based on these results, we include communication preference but not the other background variables in the following analyses.

Interest in Sound Categories. To more specifically understand what sounds may need to be prioritized in a mobile or wearable sound awareness system, participants rated the importance of eight categories of sound, which were selected based on prior work [3, 27, 39]. As shown in Figure 3a, urgent alerts (e.g., alarms, safety-critical sounds) and voices directed at you received the highest priority, both with median ratings of "extremely interested." Non-urgent alerts (e.g., non-safetycritical public announcement) and noises indicating the presence of people (e.g., paper rustling, footsteps, typing) were also deemed important, with median ratings of "very interested." We thus confirm and extend past findings [3, 27, 39] on the importance of these two categories (urgent alerts and presence of people), by providing analysis of a larger sample and analyzing their relative ordering of importance. The remaining sound categories received median ratings of "somewhat" or "slightly interested," indicating that they are potential candidates for exclusion if only a few sounds can be incorporated into a sound awareness system.
To determine whether these differences were significant and whether people with different communication preferences were interested in different sounds, we conducted a $3 \times 8$ (communication group $\times$ sound type) ANOVA with ART; these analyses go beyond results reported in previous sound interest studies [3, 39]. Both main effects and the interaction effect were significant (communication preference: $F_{2,198}=34.7, p<.001$; sound category: $F_{7,1386}=146.0$, $p<.001$; communication preference $\times$ sound category: $\left.F_{14,1386}=3.7, p<.001\right)$. Posthoc pairwise comparisons using Mann Whitney U tests with a Holm-Bonferroni adjustment were used to compare communication preference groups within each sound category. The oral group was significantly more interested in sound awareness than the sign group for all sound categories (all eight comparisons $p<.05$ ), while there were not as many differences between the oral or sign groups and the group preferring both oral and signed communication (7/16 pairwise comparisons were $p<.05)$.

When asked an open-ended question about what sounds would be most useful, participants most commonly referred to safety-related sounds, such as fire alarms, sirens, or cars approaching $(36.3 \%, N=73)$. Other emergent categories mentioned by at least $10 \%$ of participants included sounds meant to capture one's attention (e.g., someone calling, door knock), sounds indicating the presence of other people (e.g., footsteps, people talking), and direct conversations (e.g., speech). These findings confirm those of past, smaller studies [3, 27, 39].

Interest in Sound Characteristics. Participants also rated their interest in five sound characteristics: loudness, source, direction, duration, and pitch pattern (e.g., high pitch or falling pitch); Figure 3b. Source and direction had the highest interest levels with median ratings (both "very interested") and average ratings $(M=4.0, S D=1.0$ and $M=4.0, S D=1.1)$, respectively. Pitch pattern also had a median rating of "very interested" but the average indicated lower priority by many participants $(M=3.4, S D=1.3)$. As shown below, the interest in source and direction was in general significantly higher than the other sound characteristics, suggesting that a sound awareness system may not need to include the other characteristics.

Interest ratings were significantly impacted by both communication preference and sound characteristic, as shown by a $3 \times 5$ (communication group $\times$ sound characteristic) ANOVA with ART showed that (main effect communication preference: $F_{2,198}=27.0, p<.001$; main effect of sound characteristic: $\left.F_{4,792}=71.1, p<.001\right)$. There was also a significant interaction effect, showing that the three groups felt differently about some sound characteristics $\left(F_{8,792}=2.2, p=.025\right)$. To compare sound characteristics within each communication group, we conducted posthoc pairwise comparisons using 


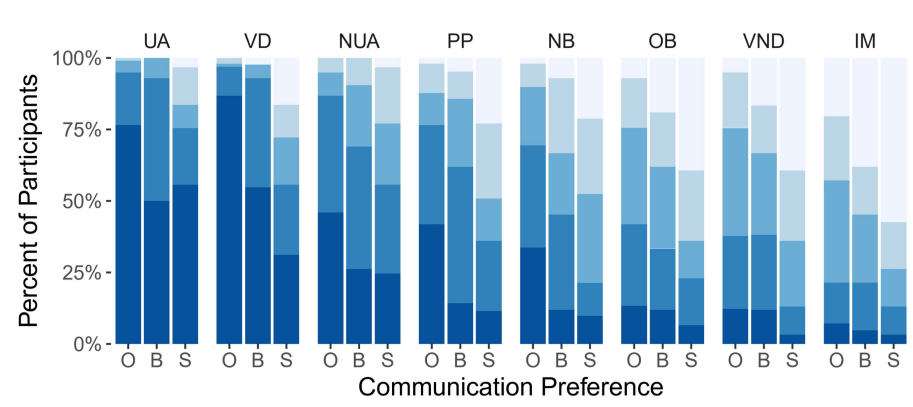

(a) Sound Categories

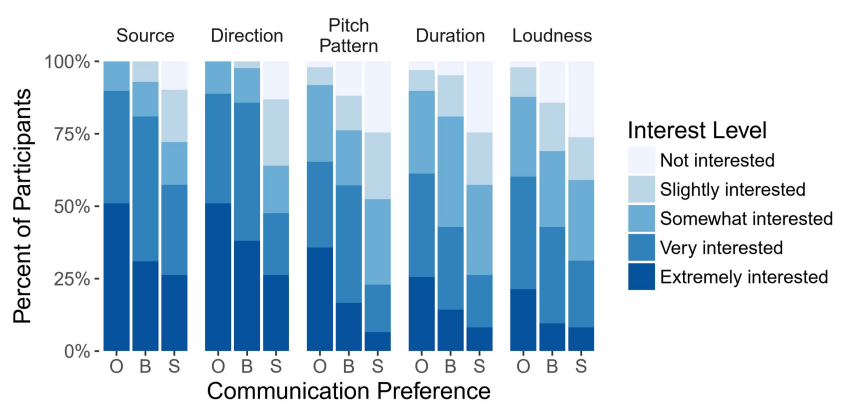

(b) Sound Characteristics

Figure 3: Interest in (a) sound categories and (b) sound characteristics by communication preference, in descending order of interest. Communication preference: $\mathrm{O}=$ oral, $\mathrm{B}=$ both, $\mathrm{S}=$ sign. Sound categories: UA = urgent alerts, VD = voices directed at you, NUA = non-urgent alerts, $\mathrm{PP}=$ presence of people, $\mathrm{NB}=$ nature background, $\mathrm{OB}=$ outdoor background, $\mathrm{VND}=\mathrm{voices}$ not directed at you, $\mathbf{I M}=$ indoor mechanical. $(N=201)$

Wilcoxon signed rank tests and a Holm-Bonferroni adjustment. The pattern of results emphasizes the importance of sound source and direction compared to the other sound characteristics. Ratings for these two characteristics were significantly different from all three other characteristics for the oral group $(p<.05)$ and for five of the six pairwise comparisons to other characteristics for the both (sign + oral) group. For the sign group, sound source was significantly different from duration and pitch pattern $(p<.05)$, with no other differences, suggesting that pitch pattern is particularly uninteresting to this group.

\section{Interest in Captions and Speech}

Past work has shown that many DHH users are interested in captions for oral conversations [16], yet full captions could be distracting and result in information overload. For some speechreaders, extracting and displaying only keywords rather than full captions may still help bootstrap their ability to follow or participate in a conversation. As an initial exploration, we asked participants about their interest in and preferences for full captions as well as keywords.

As shown in Figure 4, most participants (70.6\% of 201) were "very" or "extremely" interested in having full captions of conversations available on a wearable sound awareness device, including $70.5 \%(N=43 / 61)$ of participants who preferred sign language as their communication medium; only 7.0\% were "not interested." Interest in only keyword captions was lower, with $30.4 \%$ (of 201) being "very" or "extremely" interested, and $20.9 \%$ being "not interested".

A $2 \times 3$ (caption type $\times$ communication preference) ANOVA with ART showed that the difference between full caption and keyword interest was significant (main effect of caption type: $F_{1,198}=178.63, p<.001$ ). While the main effect of communication preference was not significant, there was a

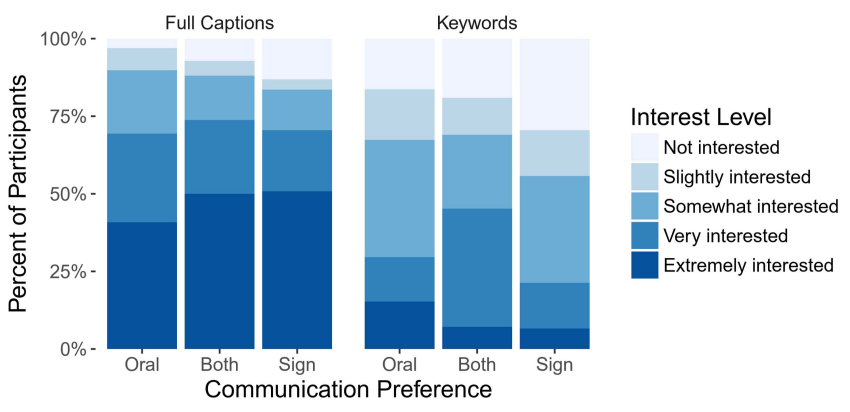

Figure 4: Interest in captions versus full keywords, captions were of significantly higher interest and interest differed by communication preference. $(N=201)$

significant interaction effect $\left(F_{2,198}=6.09, p=.003\right)$, showing that people within different communication groups felt differently about the utility of captions vs. keywords. Oral communicators tended to be less "extremely interested" in full captions and more "extremely interested" in keyword captions than the other two groups.

When asked an open-ended question about what keywords would be most useful to show, the two most common suggestions were to show the main topic/subject $(44.3 \%, N=$ $89)$ followed by proper names $(12.9 \%, N=26)$. Thirty-three participants (16.4\%), however, used the write-in opportunity to reemphasize that a "keywords only" approach would not be useful to them. For example, this participant stated: "Keywords will not give me full impression of what was spoken. This can cause confusion, misleading thoughts/information" (P149, profoundly deaf and preferred both sign and oral communication).

Summary. Most participants were "very" or "extremely" interested in sound awareness, although interest level differed based on in-person communication preferences. Past work 

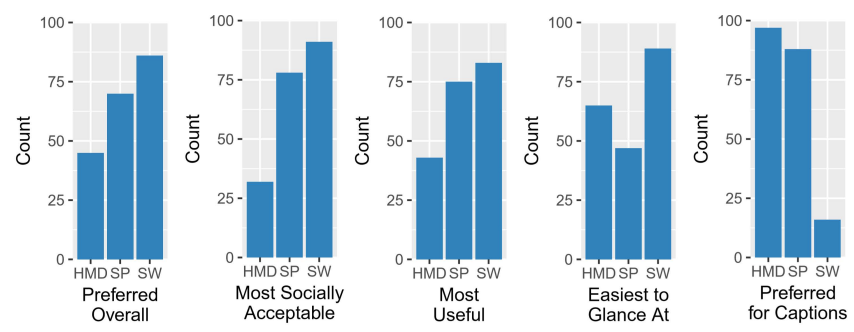

Figure 5: Counts for responses to the three form factors on a variety of measures. HMD = head-mounted display, $\mathbf{S P}=$ smartphone, and SW = smartwatch. $(N=201)$

has only examined a binary identity distinction of Deaf/deaf vs. hard of hearing [3], whereas our three communication groups show that preference is more complex-the group who preferred both sign and oral communication tended to fall between the oral-only and sign-only groups in terms of preference. We also showed that keyword captions are not of as high interest as full captions, although for some users (particularly oral communicators) they may be worth exploring. Our other findings largely confirm and extend past work [3, 27, 39] on interest in sound types (e.g., urgent sounds and voices directed at you are of high interest) and sound characteristics (e.g., direction and source are of high interest). However, due to a larger sample size and non-binary measures, we are also able to provide more detailed prioritizations of sounds. For example, nature background noises (e.g., bird chirp, wind) may be of interest even if outdoor background noises more generally are not.

\section{Form Factors and Other Design Considerations}

We compare the three mobile and wearable form factorssmartphone, smartwatch, and HMD-in terms of initial preferences and ability to support different types of sounds, as well as examine the design question of whether to show sensed sounds on demand vs. immediately.

Overall Comparison of Form Factors. When asked which of the three devices participants would prefer overall for sound awareness, results were mixed (Figure 5). The most common response was the smartwatch, although it was still selected by less than half of participants: $42.8 \%(N=86)$. compared with $34.8 \%(N=70)$ for the smartphone and $22.4 \%$ $(N=45)$ for the HMD. Still, the smartwatch's positive response is counter to recent research that has focused on smartphones [3, 39]. Preference patterns were similar for questions of social acceptability and overall use. However, the HMD performed relatively better in terms of being easy to glance at $(32.3 \%, N=65)$ and was the most preferred device for showing captions ( $48.3 \%, N=97)$, followed closely by the smartphone $(43.4 \%, N=88)$.

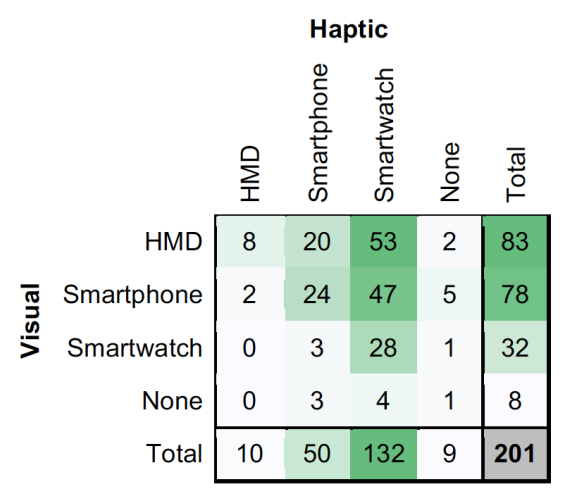

Figure 6: Responses to a question asking about ideal device setup and feedback modalities, where participants could choose visual and/or haptic on the HMD, smartwatch, smartphone, or indicate that they were not interested in a visual or vibrational feedback at all. $(N=201)$

In a follow-up open-ended question asking why participants had chosen a particular device for captions, the most common response for the HMD was to maintain eye contact or line of sight $(70.1 \%, N=68 / 98)$. Eye contact was seen as positively impacting social acceptability, mentioned by $20.0 \%(N=19)$ of those preferring the HMD, for example: "making the speaker feel that you are interested and paying attention" (P197) and "would be more natural to look at during conversation and probably most socially acceptable" (P6). The 88 participants who preferred the smartphone chose it primarily for ease $(38.6 \%, N=34)$ and the relatively large display size (37.5\%, $N=33)$. For example, "Everyone has a phone so its [sic] not distracting to pull it out. Plus, reading captions on a screen is easier than reading on a watch." (P192) and "Larger screen, ease of visibility" (P66). Finally, only 16 participants preferred the smartwatch for captions, primarily citing ease $(37.5 \%, N=6)$ and social acceptability $(18.8 \%$, $N=3)$.

When asked to create their ideal setup that could include visual and/or vibrational feedback on a smartphone, smartwatch, and/or HMD, participants chose the combinations shown in Figure 6. This data provides more nuance than the earlier ratings about overall form factor preference. First, almost all participants $(92.0 \%)$ wanted both visual and haptic feedback, emphasizing the importance of including both modalities in future designs. Second, the smartwatch was vastly preferred for haptic feedback, but most commonly in conjunction with an HMD $(N=53)$ or smartphone $(N=47)$ for visual feedback.

Sound Filtering and Immediate vs. On-demand Feedback. A sound sensing system that presents all sounds to users may overwhelm them. Even in a quiet office, for example, an air 
conditioner may be humming, people can be typing, coughing, or rustling paper, and chairs can be creaking. To begin to understand this issue, we asked if a sound awareness device should filter out some sounds and whether sounds should be shown immediately vs. on demand.

Most participants $(63.2 \%, N=127)$ wanted sound filtering, many of whom also provided open-ended rationale for that preference. Of the 107 relevant rationales, $63.6 \%(N=68)$ mentioned filtering ambient sounds, such as: "General traffic noise if in city" (P52), "don't need hums of ref [refrigerator] or coffee machine" (P78), and "background noises like bar chatter, chairs moving, papers shuffling" (P97). The importance of being able to control what sounds are filtered and/or when the filtering is turned on was also mentioned by $25.5 \%$ ( $N=$ 21) of these 107 responses. Other less frequent suggestions included filtering indirect voices and loud sounds (mentioned in fewer than $10 \%$ of the responses).

When asked about whether sounds should be displayed immediately or on demand, about half of the participants $(49.8 \%, N=100)$ preferred that all sounds be shown immediately. Most other participants $(35.5 \%, N=71)$ wanted both, based on the sound's category. The remaining few participants wanted sounds only "on demand, for example, when you press a button on the device" $(13.4 \%, N=27)$, or, for three participants, wanted sounds simply to be stored and available for later review. Overall, these findings suggest that a mobile or wearable sound awareness system should be able to show sounds immediately but also allow the user to configure some sounds to be on demand only.

Summary. Overall, the smartwatch was the most preferred form factor for a mobile/wearable sound awareness device, followed by smartphone, then HMD, although none of these devices captured a vote majority. For captions specifically and visual information in general, an HMD was the most preferred, followed by the smartphone. Almost all participants included both visual and haptic feedback when asked to specify their ideal mobile/wearable design and most (74.6\%) chose separate devices for the visual and haptic feedbackthe smartwatch being the most common choice for haptic feedback. Most participants wanted some sounds filtered out, and the plurality wanted sensed sounds to be shown immediately. Diverse preferences on these factors point to the need for customization or easy toggles.

\section{Utility and Comfort Across Social Contexts}

After asking participants to consider various aspects of mobile and wearable sound awareness devices, the survey closed with questions about utility and social acceptability (as opposed to the questions specific to form factors earlier). Figures 6 and 7 show these measures across four social contexts that offer different degrees of social distance from the

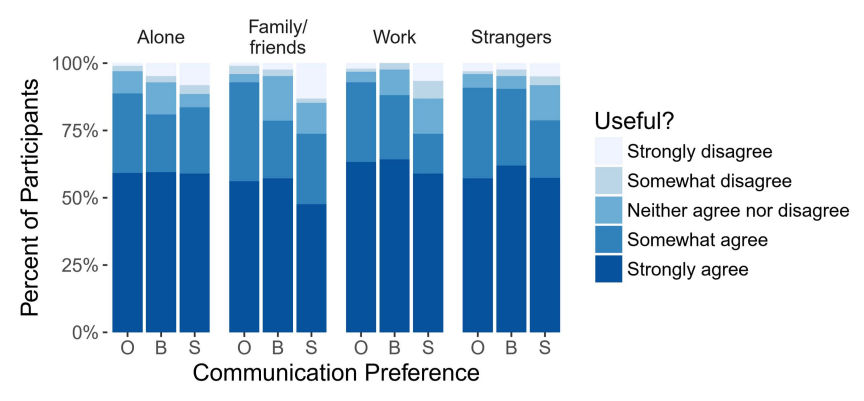

Figure 7: Usefulness ratings of a sound awareness device across social contexts, showing some variation based on context and communication preference but in general positive ratings about usefulness. $\mathbf{O}=$ oral, $\mathbf{S}=$ sign, $\mathbf{B}=$ both. $(N=201)$

user/wearer: alone, with close family and friends, at work, and with strangers. Because social acceptability and usefulness in social settings may differ for individuals who are part of Deaf culture compared to those who are not, we included communication preference in this analysis. We conducted separate $3 \times 4$ (communication group $\times$ social context) ANOVAs with ART for the two dependent variables: usefulness and social acceptability.

For usefulness, communication preference and context both influenced ratings. There were significant main and interaction effects (communication group: $F_{2,198}=5.5, p=$ .005 ; context: $F_{3,594}=6.6, p<.001$; group $\times$ context: $F_{2,198}=$ $5.8, p<.001)$. However, no posthoc pairwise comparisons on the interaction effect were significant after a Holm-Bonferroni adjustment. Based on Figure 7 and as would be expected, signers may have felt the device would be less useful in the specific context of close family/friends; qualitative comments below support this possibility.

Context also significantly affected social acceptability (main effect of context on social acceptability ratings: $F_{3,594}=11.8$, $p<.001)$. Figure 8 shows a drop in acceptability as social distance increases, which was confirmed via pairwise comparisons of social contexts: participants felt significantly less comfortable with using a sound awareness device around strangers as opposed to while alone, with family/friends, or with work colleagues ( $p<.05$ for these three comparisons). Neither the main effect of communication group on social acceptability nor the interaction effect were significant.

When asked an open-ended question about whether "where you are or who you're with" would affect the participant's willingness to use the device, $50.2 \%(N=101)$ affirmed that context would matter, 31.3\% $(N=63)$ felt that it would not matter, and the remainder were unsure or provided an answer that was coded as irrelevant. Several comments from those who felt that context would matter provided explanations that reflect the quantitative analysis above, emphasizing the potential discomfort in using the device around 


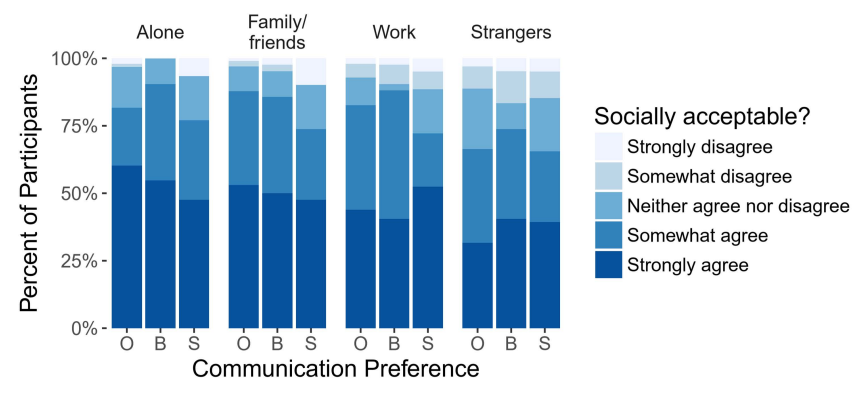

Figure 8: Social acceptability ratings across social contexts, showing decreasing acceptability as social distance increases. $\mathbf{O}=$ oral, $\mathbf{S}=$ sign, $\mathbf{B}=$ both.$(N=201)$

strangers, such as: "Most definitely. I would feel the need to explain why I have the device when I'm around someone I don't know" (P58, male, age 46, sign and oral communication). As another example, P86 (female, age 21, sign language) wrote:

"Yes. It may be distracting to strangers and [t]hey may ask about the device or may walk away when they see it because they will think that I'm not paying attention to them. With friends, family, and coworkers, however, I can explain to them the first time and then they should be comfortable with it because it is necessary for me to use in social settings."

At the same time, some saw the value of the device being higher with unfamiliar conversation partners compared to friends and family, which introduces a tension. For example, P164 (male, 45, oral communication) said, "Might not need as much with friends and family. May need more in work meetings, and most in unfamiliar situations, such as running errands, traveling." The sentiment that sound awareness and/or communication around friends and family could be supported in other ways was also reflected by other participants, such as P125 (female, age 32, sign language): "with family/friends, they will notify you anyways, so such devices are redundant." This finding reflects that in Deaf culture, direct communication and notification usually have less friction and are more personal and meaningful than technologically aided communication (e.g., tapping the table rather than texting a person to get their attention) [13].

Several responses acknowledge that this type of device would not be useful and could even be negatively received in a Deaf cultural context. For example, P96 (female, age 23, oral and sign communication) said that a device like this would not be needed "with other deaf people", but would be useful in other situations, while P95 (female, age 29, prefers sign language) emphasized this point further, stating:

"Being a member of DeafCulture, I would've thought that it'd be considered rude if I am to place sounds above visual cues available to everyone. With Hearing people, I think they'd insist on sounds being available to me."

This latter point is an example of the audism phenomenon, to describe audio-centric assumptions (i.e., based on hearing and speaking) and attitudes of supremacy [1].

Other concerns included issues of privacy, being rude, and the effort required to explain to others what the device is doing. Issues specifically related to workplace environments were also mentioned, such as devices not being allowed due to security considerations and a work environment that is not conducive to the individual using accommodations.

Conversely, participants who reported that context would not affect willingness to use the device were often less concerned with how others perceived them: "No, I am past that point in my life to care what other people thing [sic]. Also it would make me feel more comfortable in my environment" (P7, male, age 41, oral communication), "No- if anyone has an issue with it- too bad!" (P149, female, age 39, sign and oral communication), and, "No. technology like this is common today" (P153, female, age 40, prefers sign language). This lattermost quote also highlights the value of mainstream devices, particularly the smartphone and smartwatch.

Summary. We found that social contexts influence both the perceived usefulness and social acceptability of mobile and wearable sound awareness systems. The presence of strangers, in particular, correlated with decreased social acceptability. Such technology may be less useful and potentially even negatively received in a Deaf cultural context.

\section{DISCUSSION}

Sound awareness is overall of high interest to most DHH individuals, although that interest is modulated by communication preference-increasing from individuals who prefer sign language to those who prefer oral and signed communication to those who prefer oral communication. While communication preference was the most salient predictor variable in our data, age is another consideration in that people who experience hearing loss later in life have relied on sound for decades, and are less likely to know sign language and be integrated into the Deaf community. Below, we reflect on the general focus on sound awareness, and discuss design recommendations, cultural and social considerations, and limitations of our study.

\section{Why Sound Awareness?}

While our focus has been on sound awareness, sound is only one means of sensing the environment around us. Sound has an advantage over visual information in that it does not require line of sight-for example, a dripping tap in the next room can be detected remotely. A sound awareness system 
thus has the potential to provide $\mathrm{DHH}$ individuals with information that is already available to hearing people, which is an equitable access argument mentioned by participants. At the same time, other sensing approaches may more effective for some types of environmental awareness, such as movement sensors to detect general human activity. Future work should also explore user reactions to the idea of superhuman sound awareness capabilities, that is, sound sensing that exceeds the capabilities of typical hearing individuals.

\section{Design Recommendations}

A primary outcome of this work is a set of recommendations for the design of mobile and wearable sound awareness technologies. While participants only envisioned how these technologies might work and did not use them directly, the recommendations should guide further research. In addition to the recommendations below, any mobile or wearable sound awareness technology should complement and not interfere with existing visual communication strategies.

Form factor and feedback modalities. As a single device, the smartwatch is a promising form factor. However, there is a strong desire for both visual and haptic feedback ( $92 \%$ of participants) and even for this feedback to be provided on two separate devices (75\% of participants); the most promising combinations are haptic feedback on a smartwatch and visual feedback on an HMD or smartphone. For oral conversation support, HMDs offer the most promise.

Sound types. Urgent, safety-related sounds and voices directed at the user are of highest priority, followed by nonurgent alerts, people's presence, and nature background noises. Outdoor background noises, voices not directed at the user, and indoor mechanical noises are of lower interest and could be excluded or at least automatically filtered out. This recommendation reflects past work [3, 27, 39] but with a more detailed prioritization of sound categories.

Sound characteristics. For information about the characteristics of sound, the source and location are of high interest (also reflecting past work [27]).

Captions. When comparing full vs. summary captions, full captions were of highest interest, though keyword captions may be useful to some users who prefer oral communication. Captions should be provided on an HMD or smartphone.

Notification and filtering. Notifications should typically be shown immediately, but with the option to have lower priority sounds displayed only on demand. Users may want to specify which sounds should be filtered/shown and this may differ based on context (e.g., at home, at the store).

"Airplane" mode. Notifications should be able to be turned off easily, to reduce distraction and to accommodate the user's desire to act appropriately in varying social contexts.

\section{Cultural and Social Considerations}

Social context affects perceived usefulness and comfort with using a mobile or wearable sound awareness device, likely reflecting a variety of factors such as social norms around polite/rude technology use, changing social perceptions of wearable technology [21], and the stigma that can be associated with assistive technologies [38]. Several participants reported feeling the need to explain what the technology does so that other people are accepting of it, which reflects past work showing that wearable devices are more likely to be socially acceptable if they are perceived as being used for assistive purposes [35]. An important tension to address in future work is that users may be less socially comfortable using these devices around strangers, but that is also the context in which need or utility may be highest.

An additional critical aspect is how a device like this may be used (or not) in a Deaf cultural context. The significant differences between individuals preferring sign language vs. oral communication vs. both likely reflect this consideration. Deaf people often get frustrated with research that uses visual/haptic devices to support communication or notification, but that does not capture usable information at all (e.g., hearing or signing gloves), have too much friction for usable communication, or are too cognitively taxing.

\section{Limitations}

As an online survey, we were able to solicit input from a large sample of participants, but this method did not allow participants to experience what it would be like to use a sound awareness device. We expect that some preferences may change with use of a fully functional mobile or wearable system, due to issues such as information overload and accuracy of sensing; this latter issue did not arise in our study but will impact the utility of a sound awareness system and will need to be considered in the design process. Additionally, the participant background data on hearing loss was self-reported and may not be accurate. The reading level of the survey was calculated as 7th grade using the Flesch Reading Ease scale, although many questions included redundant information to aid comprehension (see supplementary materials). Finally, sound sensing (e.g., separation and identification) is an active area of research, particularly with overlapping sounds and background noise [7]-limitations that will need to be addressed by future sound sensing research or at least considered in the design of a sound feedback technology.

\section{CONCLUSION}

We presented an online survey with $201 \mathrm{DHH}$ participants investigating interest in sound awareness and preferences about the design of a mobile or wearable sound awareness 
technology. This study builds on past work on sound interests $[3,27,39]$ to quantitatively examine the influence of demographic factors on interest level. We also specifically examine mobile and wearable form factors and feedback modalities. Results show a strong preference for having both haptic and visual feedback, the latter particularly for captions, with the most preferred device design being haptic notifications on a smartwatch and visual information on a head-mounted display or smartphone. Finally, our findings surface important cultural and social considerations that will need to be addressed for the successful adoption of mobile or wearable sound awareness technologies, such as a hesitance to use a sound awareness device with strangers or around Deaf friends or family.

\section{ACKNOWLEDGMENTS}

We thank Sophie Tian for help with recruitment and Himanshu Zade for the scenario sketches. We also thank Kristoffer Everson, Rose Guttman, Jonathan Newth, and Emily Rosenfield for help with qualitative coding. This research was funded in part by the National Science Foundation under grant IIS-1763199, a Google Faculty Research Award, and the UW Reality Lab.

\section{REFERENCES}

[1] Beth S. Benedict and Marilyn Sass-Lehrer. 2007. Deaf and hearing partnerships: Ethical and communication considerations. American annals of the deaf 152, 3 (2007), 275-282.

[2] Kerry Bodine and Francine Gemperle. 2003. Effects of functionality on perceived comfort of wearables. Proceedings of the Seventh IEEE International Symposium on Wearable Computers (ISWC'03) (2003), 57-60. https://doi.org/10.1109/ISWC.2003.1241394

[3] Danielle Bragg, Nicholas Huynh, and Richard E. Ladner. 2016. A Personalizable Mobile Sound Detector App Design for Deaf and Hard-ofHearing Users. In Proceedings of the 18th International ACM SIGACCESS Conference on Computers and Accessibility. ACM Press, New York, New York, USA, 3-13. https://doi.org/10.1145/2982142.2982171

[4] Mohammad I Daoud, Mahmoud Al-Ashi, Fares Abawi, and Ala Khalifeh. 2015. In-house alert sounds detection and direction of arrival estimation to assist people with hearing difficulties. In Computer and Information Science (ICIS), 2015 IEEE/ACIS 14th International Conference on. IEEE, 297-302. https://doi.org/10.1109/ICIS.2015.7166609

[5] Harvey Dillon. 2008. Hearing aids. Hodder Arnold.

[6] Karyn L. Galvin, Jan Ginis, Robert S. Cowan, Peter J. Blamey, and Graeme M. Clark. 2001. A Comparison of a New Prototype Tickle TalkerâD́ć with the Tactaid 7. Australian and New Zealand fournal of Audiology 23, 1 (2001), 18-36.

[7] Jort F. Gemmeke, Daniel P. W. Ellis, Dylan Freedman, Aren Jansen, Wade Lawrence, R. Channing Moore, Manoj Plakal, and Marvin Ritter. 2017. Audio set: An ontology and human-labeled dataset for audio events. In Acoustics, Speech and Signal Processing (ICASSP), 2017 IEEE International Conference on. IEEE, 776-780. https://doi.org/10.1109/ ICASSP.2017.7952261

[8] Abraham Glasser, Kesavan Kushalnagar, and Raja Kushalnagar. 2017. Deaf, hard of hearing, and hearing perspectives on using automatic speech recognition in conversation. In Proceedings of the 19th International ACM SIGACCESS Conference on Computers and Accessibility.
ACM, 427-432. https://doi.org/10.1145/3132525.3134781

[9] Benjamin M Gorman. 2014. VisAural: a wearable sound-localisation device for people with impaired hearing. In Proceedings of the 16th international ACM SIGACCESS conference on Computers \& accessibility. ACM, 337-338. https://doi.org/10.1145/2661334.2661410

[10] Richard S Hallam and Roslyn Corney. 2014. Conversation tactics in persons with normal hearing and hearing-impairment. International journal of audiology 53, 3 (mar 2014), 174-81. https://doi.org/10.3109/ 14992027.2013.852256

[11] Eric G Hintz, Michael D Jones, M Jeannette Lawler, Nathan Bench, and Fred Mangrubang. 2015. Adoption of ASL classifiers as delivered by head-mounted displays in a planetarium show. fournal of Astronomy \& Earth Sciences Education (FAESE) 2, 1 (2015), 1-16.

[12] F Wai-ling Ho-Ching, Jennifer Mankoff, and James A Landay. 2003. Can You See What I Hear?: The Design and Evaluation of a Peripheral Sound Display for the Deaf. In Proceedings of the SIGCHI Conference on Human Factors in Computing Systems (CHI '03). ACM, New York, NY, USA, 161-168. https://doi.org/10.1145/642611.642641

[13] Thomas K Holcomb. 2012. Introduction to American deaf culture. Oxford University Press.

[14] Sture Holm. 1979. A simple sequentially rejective multiple test procedure. Scandinavian journal of statistics (1979), 65-70.

[15] Daniel J. Hruschka, Deborah Schwartz, Daphne Cobb St. John, Erin Picone-Decaro, Richard A. Jenkins, and James W. Carey. 2004. Reliability in Coding Open-Ended Data: Lessons Learned from HIV Behavioral Research. Field Methods 16, 3 (aug 2004), 307-331. https: //doi.org/10.1177/1525822X04266540

[16] Dhruv Jain, Leah Findlater, Christian Volger, Dmitry Zotkin, Ramani Duraiswami, and Jon Froehlich. 2015. Head-Mounted Display Visualizations to Support Sound Awareness for the Deaf and Hard of Hearing. In Proceedings of the 33rd Annual ACM Conference on Human Factors in Computing Systems. 241--250. https://doi.org/10.1145/2702123. 2702393

[17] Dhruv Jain, Rachel Franz, Leah Findlater, Jackson Cannon, Raja Kushalnagar, and Jon Froehlich. 2018. Towards Accessible Conversations in a Mobile Context for People who are Deaf and Hard of Hearing. In Proceedings of the International Conference on Computers and Accessibility (ASSETS). ACM.

[18] Dhruv Jain, Angela Carey Lin, Marcus Amalachandran, Aileen Zeng, Rose Guttman, Leah Findlater, and Jon Froehlich. 2019. Exploring Sound Awareness in the Home for People who are Deaf or Hard of Hearing. In SIGCHI Conference on Human Factors in Computing Systems (CHI). ACM.

[19] Michael Jones, M Jeannette Lawler, Eric Hintz, Nathan Bench, Fred Mangrubang, and Mallory Trullender. 2014. Head Mounted Displays and Deaf Children: Facilitating Sign Language in Challenging Learning Environments. In Proceedings of the 2014 Conference on Interaction Design and Children (IDC '14). ACM, New York, NY, USA, 317-320. https://doi.org/10.1145/2593968.2610481

[20] Y Kaneko, Inho Chung, and K Suzuki. 2013. Light-Emitting Device for Supporting Auditory Awareness of Hearing-Impaired People during Group Conversations. In Systems, Man, and Cybernetics (SMC), 2013 IEEE International Conference on. 3567-3572. https://doi.org/10.1109/ SMC.2013.608

[21] Norene Kelly. 2017. All the world's a stage: what makes a wearable socially acceptable. interactions 24,6 (2017), 56-60. https://doi.org/10. $1145 / 3137093$

[22] Ki-Won Kim, Jung-Woo Choi, and Yang-Hann Kim. 2013. An Assistive Device for Direction Estimation of a Sound Source. Assistive Technology 25, 4 (oct 2013), 216-221. https://doi.org/10.1080/10400435.2013. 768718 
[23] Raja S Kushalnagar, Gary W Behm, Joseph S Stanislow, and Vasu Gupta 2014. Enhancing caption accessibility through simultaneous multimodal information: visual-tactile captions. In Proceedings of the 16th international ACM SIGACCESS conference on Computers \& accessibility. ACM, 185-192. https://doi.org/10.1145/2661334.2661381

[24] James Lee. 1997. Speechreading in Context: A Guide for Practice in Everyday Settings. Ph.D. Dissertation. Laurent Clerc National Deaf Education Center, Gallaudet University.

[25] Deborah Lupton and Wendy Seymour. 2000. Technology, selfhood, and physical disability. Social Science \& Medicine 50 (2000), 1851-1862. https://doi.org/10.1016/S0277-9536(99)00422-0

[26] Marc Marschark, Harry G Lang, and John A Albertini. 2001. Educating deaf students: From research to practice. Oxford University Press.

[27] Tara Matthews, Janette Fong, F. Wai-Ling Ho-Ching, and Jennifer Mankoff. 2006. Evaluating non-speech sound visualizations for the deaf. Behaviour \& Information Technology 25, 4 (jul 2006), 333-351. https://doi.org/10.1080/01449290600636488

[28] Tara Matthews, Janette Fong, and Jennifer Mankoff. 2005. Visualizing non-speech sounds for the deaf. In Proceedings of the 7th international ACM SIGACCESS conference on Computers and Accessibility - Assets '05. ACM Press, New York, New York, USA, 52-59. https://doi.org/10. 1145/1090785.1090797

[29] Matthias Mielke and Rainer Brück. 2015. A Pilot Study about the Smartwatch as Assistive Device for Deaf People. In Proceedings of the 17th International ACM SIGACCESS Conference on Computers \& Accessibility. ACM, 301-302. https://doi.org/10.1145/2700648.2811347

[30] Matthias Mielke and Rainer Brueck. 2015. Design and evaluation of a smartphone application for non-speech sound awareness for people with hearing loss. In Engineering in Medicine and Biology Society (EMBC), 2015 37th Annual International Conference of the IEEE. IEEE, 5008-5011. https://doi.org/10.1109/EMBC.2015.7319516

[31] Donald F Moores. 2010. The history of language and communication issues in deaf education. The Oxford handbook of deaf studies, language, and education 2 (2010), 17-30.

[32] National Institute on Deafness and Other Communication Disorders (NIDCD). 2014. Cochlear Implants. http://www.nidcd.nih.gov/health/ hearing/pages/coch.aspx

[33] Becky Sue Parton. 2016. Glass Vision 3D: Digital Discovery for the Deaf. TechTrends (2016), 1-6.

[34] Yi-Hao Peng, Ming-Wei Hsu, Paul Taele, Ting-Yu Lin, Po-En Lai, Leon Hsu, Tzu-chuan Chen, Te-Yen Wu, Yu-An Chen, Hsien-Hui Tang, and Mike Y. Chen. 2018. SpeechBubbles: Enhancing Captioning Experiences for Deaf and Hard-of-Hearing People in Group Conversations. In SIGCHI Conference on Human Factors in Computing Systems (CHI). ACM, Paper No. 293. https://doi.org/10.1145/3173574.3173867
[35] Halley Profita, Reem Albaghli, Leah Findlater, Paul Jaeger, and Shaun Kane. 2016. The AT Effect: How Disability Affects the Perceived Social Acceptability of Head-Mounted Display Use. In Proceedings of the SIGCHI Conference on Human Factors in Computing Systems (CHI 2016). 4884-4895. https://doi.org/10.1145/2858036.2858130

[36] Halley Profita, James Clawson, Scott Gilliland, Clint Zeagler, Thad Starner, Jim Budd, and Ellen Yi-luen Do. 2013. Don't mind me touching my wrist: a case study of interacting with on-body technology in public. In Proc. ISWC'13. 89-96. https://doi.org/10.1145/2493988.2494331

[37] Ruiwei Shen, Tsutomu Terada, and Masahiko Tsukamoto. 2013. A system for visualizing sound source using augmented reality. International fournal of Pervasive Computing and Communications 9, 3 (2013), 227-242. https://doi.org/10.1108/IJPCC-07-2013-0018

[38] Kristen Shinohara and JO Wobbrock. 2011. In the shadow of misperception: assistive technology use and social interactions. SIGCHI Conference on Human Factors in HCI (2011), 705-714. https://doi.org/ 10.1145/1978942.1979044

[39] Liu Sicong, Zhou Zimu, Du Junzhao, Shangguan Longfei, Jun Han, and Xin Wang. 2017. UbiEar: Bringing Location-independent Sound Awareness to the Hard-of-hearing People with Smartphones. Proceedings of the ACM on Interactive, Mobile, Wearable and Ubiquitous Technologies 1, 2 (2017), 17. https://doi.org/10.1145/3090082

[40] Sony. [n. d.]. Sony Access Glasses. https://bit.ly/2DgwpPK

[41] Martin Tomitsch and Thomas Grechenig. 2007. Design Implications for a Ubiquitous Ambient Sound Display for the Deaf.. In Conference \& Workshop on Assistive Technologies for People with Vision \& Hearing Impairments Assistive Technology for All Ages (CVHI 2007), M.A. Hersh (Ed.).

[42] Martin Weigel, Vikram Mehta, and Jürgen Steimle. 2014. More Than Touch: Understanding How People Use Skin As an Input Surface for Mobile Computing. In Proceedings of the SIGCHI Conference on Human Factors in Computing Systems (CHI '14). ACM, New York, NY, USA, 179-188. https://doi.org/10.1145/2556288.2557239

[43] Jacob O Wobbrock, Leah Findlater, Darren Gergle, and James J Higgins. 2011. The Aligned Rank Transform for Nonparametric Factorial Analyses Using Only Anova Procedures. In Proceedings of the SIGCHI Conference on Human Factors in Computing Systems (CHI '11). ACM, New York, NY, USA, 143-146. https://doi.org/10.1145/1978942.1978963

[44] Eddy Yeung, Arthur Boothroyd, and Cecil Redmond. 1988. A Wearable Multichannel Tactile Display of Voice Fundamental Frequency. Ear and Hearing 9, 6 (1988), 342-350.

[45] Hanfeng Yuan, Charlotte M. Reed, and Nathaniel I. Durlach. 2005. Tactual display of consonant voicing as a supplement to lipreading. The fournal of the Acoustical Society of America 118, 2 (aug 2005), 1003. https://doi.org/10.1121/1.1945787 